\title{
Medium-scale traveling ionospheric disturbances by three-dimensional ionospheric GPS tomography
}

\author{
C. H. Chen ${ }^{1 *}$, A. Saito ${ }^{2}$, C. H. Lin ${ }^{1}$, M. Yamamoto ${ }^{3}$, S. Suzuki ${ }^{3}$ and G. K. Seemala ${ }^{4}$
}

\begin{abstract}
In this study, we develop a three-dimensional ionospheric tomography with the ground-based global position system (GPS) total electron content observations. Because of the geometric limitation of GPS observation path, it is difficult to solve the ill-posed inverse problem for the ionospheric electron density. Different from methods given by pervious studies, we consider an algorithm combining the least-square method with a constraint condition, in which the gradient of electron density tends to be smooth in the horizontal direction and steep in the vicinity of the ionospheric F2 peak. This algorithm is designed to be independent of any ionospheric or plasmaspheric electron density models as the initial condition. An observation system simulation experiment method is applied to evaluate the performance of the GPS ionospheric tomography in detecting ionospheric electron density perturbation at the scale size of around $200 \mathrm{~km}$ in wavelength, such as the medium-scale traveling ionospheric disturbances.
\end{abstract}

\section{Background}

Since late 1980s, the satellite radio tomography method has been employed to study the ionospheric electron density structure. Austen et al. $(1986,1988)$ first proposed the ionospheric tomography technique and measured the ionospheric total electron content (TEC) along the line of sight (LOS) from naval navigational satellite system (NNSS) to the ground-based receivers. They further successfully reconstructed the two-dimensional image of the ionospheric electron density from the onedimensional TEC data by using the technique of computerized ionospheric tomography (CIT). After that, many papers were published regarding the improvement and applications of CIT (Austen et al. 1988; Raymund et al. 1990; Yeh and Raymund 1991; Andreeva et al. 1992; Fremouw et al. 1992; Pryse and Kersley 1992; Fehmers 1994; Kunitake et al. 1995), which show the CIT has become a usable radio technique for the ionospheric electron density reconstruction (Raymund et al. 1994). Recent studies (Pryse 2003; Kersley 2005) presented that combination

\footnotetext{
*Correspondence: koichi@mail.ncku.edu.tw

${ }^{1}$ Department of Earth Sciences, National Cheng Kung University, Tainan, Taiwan

Full list of author information is available at the end of the article
}

of the CIT with ionospheric models could help further explore the variations of global/regional ionospheric electron density structures in more detail.

Previous CIT studies usually used the high-rate NNSS observation data to reconstruct the ionospheric electron density structure (Raymund et al. 1990; Kunitake et al. 1995). This kind of low earth orbit (LEO) satellites normally has relatively high ground-track velocity. Therefore, the ionospheric electron density structure can be seen as a "snapshot" for the density inversion during the satellite passing by (within 15-30 min). In the recent decades, scientists use the ground-based global position system (GPS) receivers to derive the TEC along GPS satellites to the receivers. Because GPS-TEC data have fine spatial and temporal resolution, they are used to present the two-dimensional TEC map in the longitude-latitude plane (Wilson et al. 1992; Sardon et al. 1994; Liu et al. 1996). If we employ the CIT technique to the GPS-TEC observation data, we could obtain the altitude information of ionospheric electron density profile additionally. However, due to the slow-moving ground tracks and high elevation angle of GPS satellite, the spatial distribution of GPS-TEC observation data is not enough to reconstruct the three-dimensional electron density structure if the

\section{楚 Springer}

(c) 2016 Chen et al. This article is distributed under the terms of the Creative Commons Attribution 4.0 International License (http://creativecommons.org/licenses/by/4.0/), which permits unrestricted use, distribution, and reproduction in any medium, provided you give appropriate credit to the original author(s) and the source, provide a link to the Creative Commons license, and indicate if changes were made. 
ground-based receivers are sparse. A dense GPS receiver network, such as Japan GPS Earth Observation Network (GEONET), is required to overcome the limitation.

Although there are regions of dense ground-based GPS receivers that could be chosen to perform GPS tomography, it is still difficult to straightforwardly solve the illposed inverse problem. This is due to the fact that the high orbit of GPS satellites (around 20,200 km) results in fewer low elevation LOS ray paths and thus less altitudinal information. The other problem is the lack of observations at the higher altitude region resulting in numerous areas that have no LOS ray passing through. Therefore, scientists employ an iterative method with an initial guess value from the ionospheric model, such as the international reference ionosphere (IRI) model, to approach the true solution of ionospheric electron density (Raymund 1995; Bilitza and Reinisch 2008; Ssessanga et al. 2015). As a result, the solutions might be sensitive to the initial guess. Therefore, it might not be suitable for the ionospheric disturbance period when ionospheric electron density may become irregular, such as the periods of solar flare or magnetic storm. In this study, an ionospheric GPS tomography algorithm with the constrained least-square method is developed and used to reconstruct the threedimensional electron density structure without using any initial model guess. An observation system simulation experiment (OSSE) is further employed to evaluate the performance of GPS tomography method during quiet time and disturbed ionospheric period.

\section{Methods}

The TEC value along the LOS from the GPS satellite to the ground-based receiver can be calculated by the following equation:

$$
\mathrm{TEC}=\int_{\text {rec }}^{\text {sat }} N_{\mathrm{e}} \mathrm{d} s
$$

in which $N_{\mathrm{e}}=$ electron density, $\mathrm{d} s=$ length along the radio ray path, sat $=$ location of GPS satellite, and rec $=$ location of ground-based GPS receiver. In order to inverse the three-dimensional electron density structure from the two-dimensional TEC observation, we divide the three-dimensional space into small grids with the altitude from 80 to 20,200 km (Fig. 1). Furthermore, we assume the electron density in each small grid has the same value. Therefore, the GPS-TEC observation along the ray path from a GPS satellite to a ground-based GPS receiver can be presented as:

$$
b_{i}=\sum_{j} A_{i j} x_{j}
$$

where $A_{i j}=$ length of path $i$ in grid $j, x_{j}=$ electron density in grid $j$, and $b_{i}=$ TEC value along one GPS observational

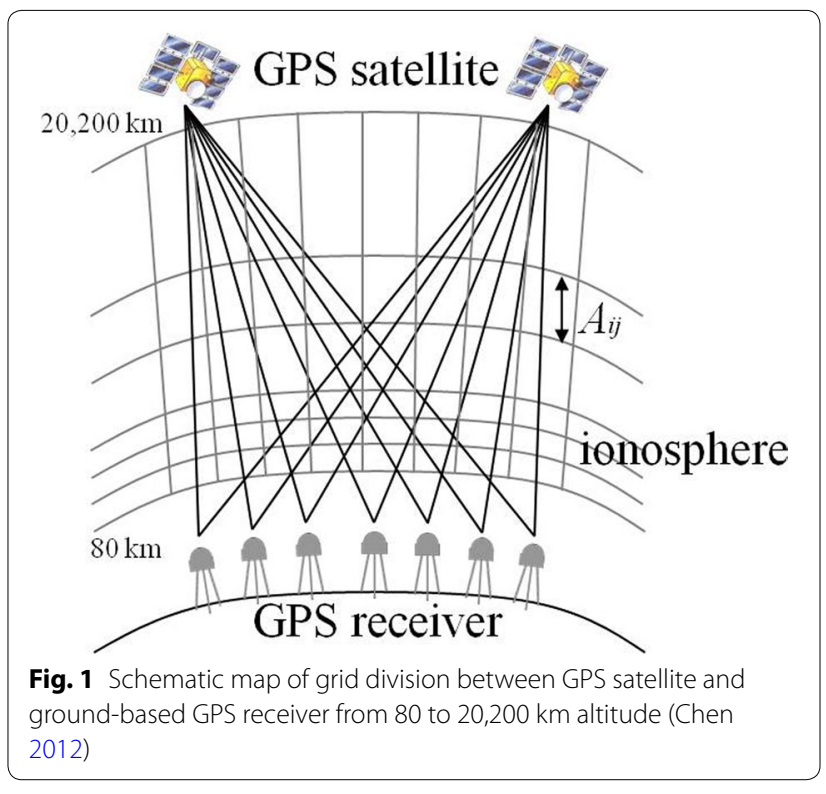

path $i$. However, due to lack of the horizontal GPS-TEC observation paths and the numerous grids in $A$ matrix that have no LOS ray passing through, the electron density ( $x$ matrix) cannot be calculated by straightforward solution of the least-square method. Here, we propose a modified methodology by applying a constrained condition in the least-square fitting to solve this ill-posed inverse problem.

The cost function and the solution (electron density, $x$ matrix in 2) of constrained least-square method are showed as:

$$
J(x)=\|b-A x\|^{2}+\lambda\|W x\|^{2}
$$

where $W x=\sum_{j=1}^{N} \sum_{k=1}^{6} C_{j k}\left(x_{j}-x_{j k}\right)$

$$
x=\left(A^{\mathrm{T}} A+\lambda W^{\mathrm{T}} W\right)^{-1} A^{\mathrm{T}} b
$$

in which $J(x)$ is the cost function, which shows the normal least-square fitting at the first term of right-hand side and the constrained condition at the second term of right-hand side. $W$ is the constraint matrix, which is a zero matrix but the value is 1 at the six neighbor grids of $x$. Therefore, $W x$ presents the total electron density differences of each grid, $x_{j}$, from its six neighbor grids, $x_{j k}$. $C$ is the matrix of constraint parameter and indicates the weighting of constrain condition which depends on the altitude. Since the electron density around the ionospheric $\mathrm{E}$ and $\mathrm{F}$ regions has diurnal variations, a relatively small constraint parameter is applied from 180 to $650 \mathrm{~km}$. Table 1 presents the values of the constraint parameters used in this paper. $\lambda$ is the regularization parameter, which is suggested by balancing the least-square term 
Table 1 Constraint parameters used in the GPS tomography

\begin{tabular}{ll}
\hline Altitude & Constraint value \\
\hline $80 \mathrm{~km}$ & $10^{-1}$ \\
$180 \mathrm{~km}$ & $10^{-3}$ \\
$650 \mathrm{~km}$ & $10^{-3}$ \\
$1000 \mathrm{~km}$ & $10^{-2}$ \\
$1500 \mathrm{~km}$ & $10^{-1}$ \\
$2000 \mathrm{~km}$ & 1 \\
$25,000 \mathrm{~km}$ & 1 \\
\hline
\end{tabular}

and the constraint condition in (3) (Seemala et al. 2014). The ionospheric GPS tomography algorithm employed in this paper is similar to that described in Seemala et al. (2014), but using the fixed constraint parameters to totally remove the model dependence and further using the smaller spatial resolution in latitude and longitude to evaluate its performance in detecting relatively smallscaled ionospheric density structure.

\section{Results for quiet ionosphere}

In this paper, we choose 1241 ground-based GPS receivers from GEONET and International GNSS Service (IGS) around Japanese region for evaluating the ionospheric GPS tomography. Figure 2 indicates the location of GPS receivers and the horizontal range of tomography. The horizontal resolution is $1^{\circ}$ in latitude and longitude within the blue area $\left(6^{\circ} \mathrm{N}-70^{\circ} \mathrm{N}\right.$ in latitude and $100^{\circ} \mathrm{E}-165^{\circ} \mathrm{E}$ in longitude). The black area $\left(6^{\circ} \mathrm{N}-54^{\circ} \mathrm{N}\right.$ in latitude and $120^{\circ} \mathrm{E}-154^{\circ} \mathrm{E}$ in longitude) has $2^{\circ}$ horizontal resolution. Outside the black area, the resolution becomes $5^{\circ}$. The vertical range of tomography is set to the altitude from 80 to $20,000 \mathrm{~km}$. The vertical resolutions are $20 \mathrm{~km}$ within $80-500 \mathrm{~km}$ range, $50 \mathrm{~km}$ within $550-900 \mathrm{~km}$ range, $100 \mathrm{~km}$ within $1000-2000 \mathrm{~km}$ range, and $5000 \mathrm{~km}$ within 5000-20,000 km range, respectively.

In order to generate the synthetic TEC observations for the OSSE, we use the ionospheric empirical model, NeQuick (Radicella and Leitinger 2001), as the model truth. Based on the LOS geometry between GPS satellites and ground-based GPS receivers, the synthetic TEC data are calculated by integrating the NeQuick electron density in each grid box along the GPS observation ray path. The synthetic GPS-TEC observations in the OSSE correspond to realistic GPS-to-receiver geometries that are collected at 10:00 UT on May 23, 2012. Furthermore, the synthetic TEC values are assumed to have no observational error in this study.

Figure 3 shows the two-dimensional electron density results of GPS tomography on altitude-latitude plane at $136^{\circ} \mathrm{E}$ longitude (Fig. 3a-c) and latitude-longitude plane at $300 \mathrm{~km}$ altitude (Fig. 3d-f), respectively. Comparing with the OSSE model truth given by NeQuick (Fig. 3a) and tomography (Fig. 3b) electron density in the altitude-latitude plane, it can be seen that the tomography results show a similar electron density distribution with the model truth, high (low) electron density at low (high) latitude region. The reconstructed electron density error shown in Fig. 3c indicates a well reproduction of OSSE model truth. It shows that the reconstructed error is located at the range of $-3.0 \times 10^{11}$ to $2.0 \times 10^{11} \mathrm{el} / \mathrm{m}^{3}$. Figure $3 \mathrm{~d}$, e shows the horizontal electron density structures at $300 \mathrm{~km}$ altitude on the latitude-longitude plane for OSSE model truth given by NeQuick and tomography result, respectively. It is clearly seen that the tomography results resemble the electron density distributions given by the model truth, indicating a good tomographic reconstruction. Their difference shown in Fig. 3f further suggests that the GPS tomography method can well reproduce the model truth, except for the region of $25^{\circ} \mathrm{N}-30^{\circ} \mathrm{N}$ and $140^{\circ} \mathrm{E}-145^{\circ} \mathrm{E}$. This discrepancy might be caused by the lower LOS ray paths around this region.

The root-mean-square error (RMSE) is further used as the accuracy measurement of the tomography reconstruction. The RMSE is defined as the root-mean-square difference between the tomography electron density and the electron density of OSSE model truth, and can be described as

$$
\mathrm{RMSE}=\sqrt{\frac{\sum_{j=1}^{N}\left(x_{j, \text { tomo }}-x_{j, \text { model }}\right)^{2}}{N}}
$$

in which $N$ is the total number of grid boxes with the radio ray path passed, $x_{j \text {,tomo }}$ is the tomography electron density in each grid box, and $x_{j \text {,model }}$ is the model truth electron density given by NeQuick. In this case, the RMSE is around $7.97 \times 10^{10} \mathrm{el} / \mathrm{m}^{3}$ and the average model truth electron density at $300 \mathrm{~km}$ altitude is around $9.83 \times 10^{11} \mathrm{el} / \mathrm{m}^{3}$. The RMSE value of the tomography is smaller than that of the average $300 \mathrm{~km}$ electron density in the ionosphere with the ratio of $8.1 \%$, which confirms the reliability of the inversion reconstruction using the GPS tomography algorithm proposed in this paper.

A histogram shown in Fig. 4a further presents the overall reconstructed error distribution of tomography electron densities difference with the model truth densities at each grid box in the defined tomography range. Results show that the error distributes like a Gaussian function. Around $66 \%$ of the tomography results are within $\pm 3.75 \times 10^{10} \mathrm{el} / \mathrm{m}^{3}$ error range, which is $\pm 3.8 \%$ of the average electron density at $300 \mathrm{~km}$ altitude. Furthermore, we compare the tomography-reconstructed vertical density profile with the OSSE model truth at three locations. These three locations are the southwest of Japan $\left(26^{\circ} \mathrm{N}\right.$, 


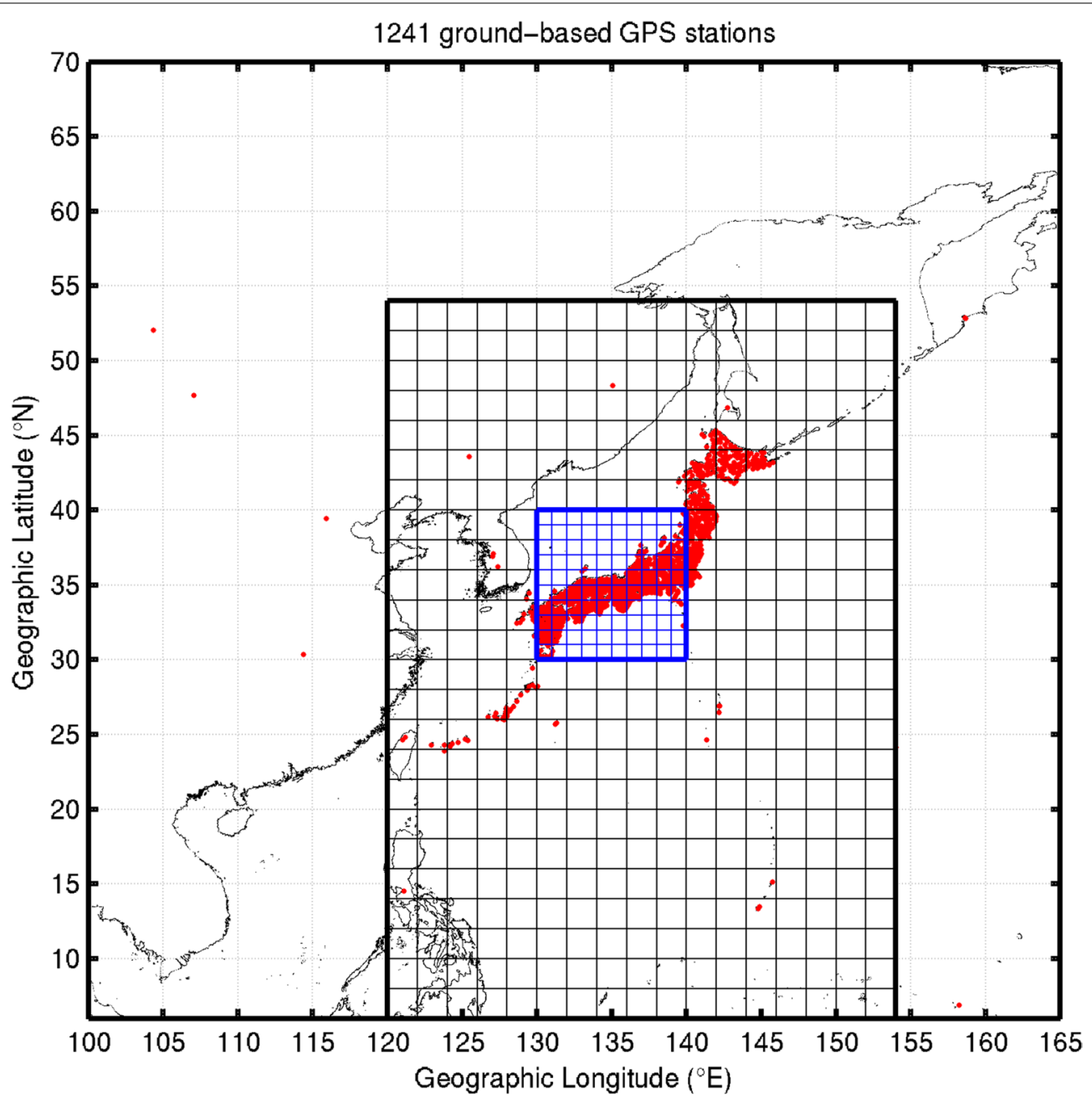

Fig. 2 Horizontal range of GPS tomography in this study. The latitude range is from $6^{\circ} \mathrm{N}$ to $70^{\circ} \mathrm{N}$ and the longitude is from $100^{\circ} \mathrm{E}$ to $165^{\circ} \mathrm{E}$. The horizontal resolutions are $1^{\circ}$ within the blue area $\left(30^{\circ} \mathrm{N}-40^{\circ} \mathrm{N}\right.$ in latitude and $130^{\circ} \mathrm{N}-140^{\circ} \mathrm{N}$ in longitude), $2^{\circ}$ within the black area $\left(6^{\circ} \mathrm{N}-54^{\circ} \mathrm{N}\right.$ in latitude and $120^{\circ} \mathrm{N}-154^{\circ} \mathrm{N}$ in longitude), and $5^{\circ}$ outside the black area, respectively. Red point indicates the location of ground-based GPS receiver

$\left.128^{\circ} \mathrm{E}\right)$, the center of Japan $\left(36^{\circ} \mathrm{N}, 136^{\circ} \mathrm{E}\right)$, and the northeast of Japan $\left(40^{\circ} \mathrm{N}, 140^{\circ} \mathrm{E}\right)$, respectively (red points in Fig. 3f). Figure $4 \mathrm{~b}-\mathrm{d}$ indicates that the reconstructed $h m F 2$ and $N m F 2$ are in good agreement with the model truth, especially above the $h m F 2$. However, the reconstructed electron densities are larger than the model truth at the bottom side of F2 peak. Furthermore, the reconstructed errors are small for the $N m F 2$ with larger error at the lower latitude region (Fig. 4b) than at the higher latitude region (Fig. 4d). The reconstructed $h m F 2$ is located at $320 \mathrm{~km}$ altitude, but the model truth of $h m F 2$ is $300 \mathrm{~km}$ altitude, which has $\sim 6.67 \%$ enhancement of $300 \mathrm{~km}$ altitude. This different $h m F 2$ altitude might be caused by the vertical resolution $(20-\mathrm{km}$ resolution around $F$ peak) of tomography in the study. The result, however, validates the performance of the GPS tomography algorithm to reconstruct the three-dimensional electron density from the two-dimensional TEC data without the ionospheric initial guess.

\section{Results for ionosphere with MSTID}

The traveling ionospheric disturbances (TIDs) are wavelike electron density disturbances that propagate through the ionosphere and cause wave-like TEC disturbances (Davies 1990; Kelley 2011) due to external energy input to the ionosphere and/or plasma instabilities. In midlatitude, medium-scale TID (MSTID) appears frequently. Its two-dimensional structure in airglow images and TEC disturbances were further observed by ground-based all-sky imager (Ogawa et al. 2002; Shiokawa et al. 2003; Otsuka et al. 2009) and dense GPS receiver network over Japan (Saito et al. 1998; Tsugawa et al. 2004; Otsuka et al. 2011), respectively. However, the TID-triggered threedimensional electron density structures are hardly known 

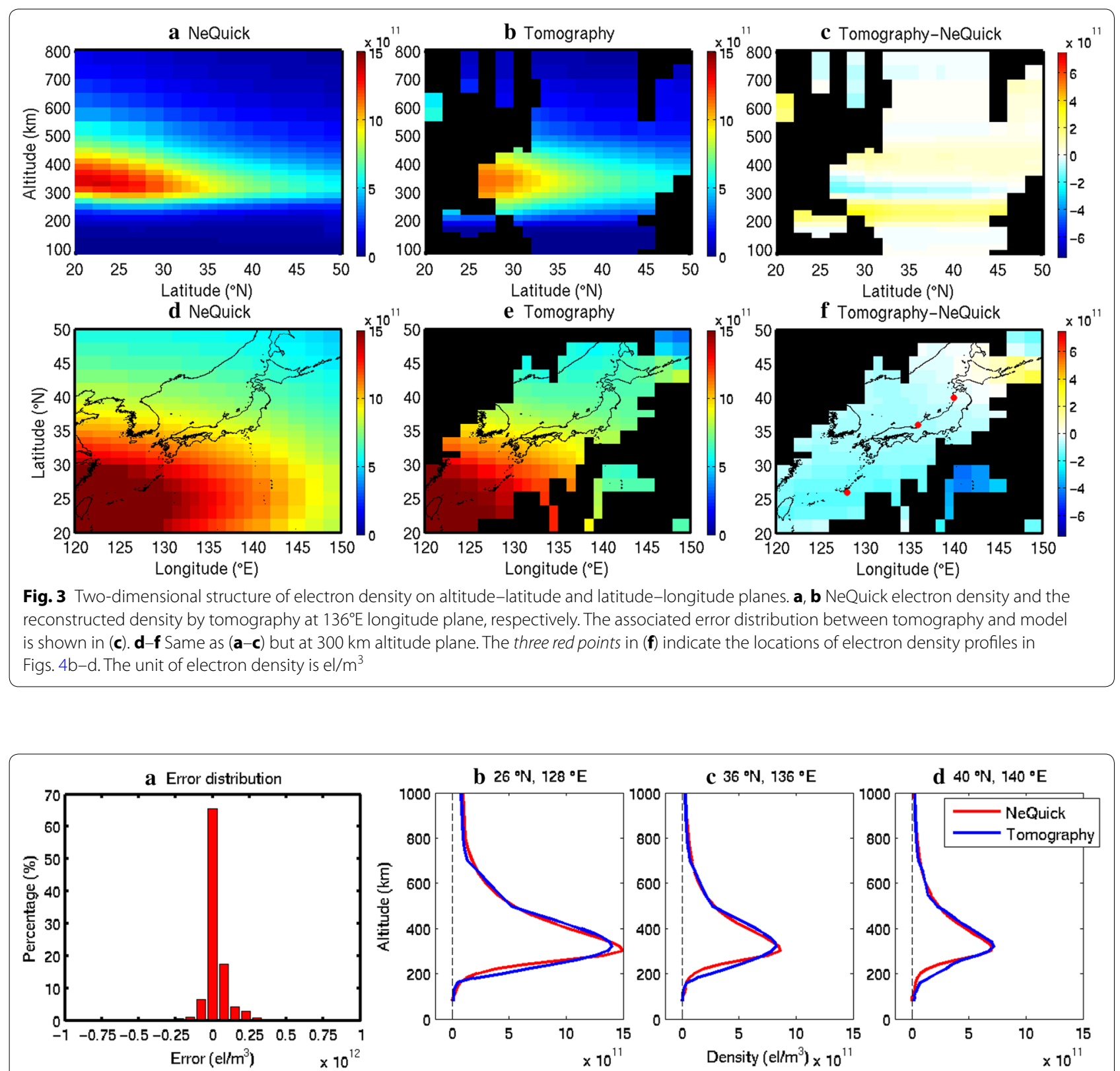

Fig. 4 Distribution of the overall reconstructed density error (a) and vertical density profile at the three locations (b-d). Blue line is the tomography density profile, and red line is the NeQuick model density

by using 630-nm all-sky images and GPS-TEC observations. The ionospheric tomography is one of the methods to reconstruct the three-dimensional density structure.

In order to evaluate whether the GPS tomography algorithm in this paper could be employed to reconstruct the ionospheric perturbations, we attempt to reproduce the three-dimensional density structure of MSTID from the two-dimensional GPS-TEC observations over Japan by performing an OSSE. Since the MSTID is defined as a medium size of TID with the horizontal wavelengths of
100-250 km (Hunsucker 1982), a density perturbation caused by electric filed during the nighttime (c.f. Otsuka et al. 2013) is applied to the quiet-ionosphere electron density for synthetic MSTID electron density as:

$$
N_{\mathrm{e}}^{\prime}=-i \omega^{-1} v^{\prime}\left[\frac{\partial N_{\mathrm{e}}}{\partial z} \cos I \sin D\right]
$$

where $v^{\prime}(=25 \mathrm{~m} / \mathrm{s})$ is the drift velocity caused by the polarization electric filed. $\omega$ is the angular frequency ( $=2 \pi / T$, where $T$ is the propagation period of $40 \mathrm{~min}$ ). $I$ 
and $D$ indicate the inclination angle $\left(=45^{\circ}\right)$ of the magnetic field line and the azimuth angle $\left(=225^{\circ}\right)$ between the MSTID propagation direction and the magnetic north, respectively. The horizontal wavelength is set as $200 \mathrm{~km}$ to match the wavelength definition of MSTID. As a result, the upward and downward motions of electron densities are observed above $30^{\circ} \mathrm{N}$ latitude (Fig. 5a). Furthermore, the wavefront of electron density perturbation is in northwest-southeast (NW-SE) direction, a typical MSTID waveform alignment, as shown in Fig. $5 \mathrm{~d}$.

Figure $5 \mathrm{~b}$ illustrates the reconstructed electron density distribution by GPS tomography as a function of altitude and latitude at the $136^{\circ} \mathrm{E}$ longitude plane. The tomography result shows several clear density perturbation regions above $30^{\circ} \mathrm{N}$ latitude and around $300 \mathrm{~km}$ altitude, which is similar to the MSTID structure from the OSSE model truth (Fig. 5a). The distribution of reconstructed electron density error also has the similar pattern with the quiet time error (Fig. 3c) and similar error range of $-2.0 \times 10^{11}$ to $3.0 \times 10^{11} \mathrm{el} / \mathrm{m}^{3}$ (Fig. $5 \mathrm{c}$ ). Figure $5 \mathrm{~d}$, e shows a comparison of the horizontal electron density structure by model truth and tomography reconstruction at $300 \mathrm{~km}$ altitude. Meanwhile, the typical NW-SE alignment of MSTIDs can be seen by the tomography reconstruction around $130-140^{\circ} \mathrm{E}$ longitude (Fig. 5e), similar to the perturbed density structure for synthetic MSTIDs (Fig. 5d). This comparison indicates that the proposed GPS tomography algorithm is capable of reconstructing ionospheric perturbation of $200 \mathrm{~km}$ scale size based on the OSSE. The RMSE is also calculated for the MSTID case. The RMSE value is around $8.61 \times 10^{10} \mathrm{el} / \mathrm{m}^{3}$, which is around $8.8 \%$ of the average electron density at $300 \mathrm{~km}$ altitude. Comparing with the RMSE of quiet time case and MSTID case, the RMSE of MSTID case is a little larger than that of quiet time case. This might be caused by the disturbance density of MSTID. However, the tomography results still reproduce the synthetic MSTID density structure well. The distribution of overall tomography inversion error is further shown in Fig. 6a. The Gaussian distribution of error shows that around $62 \%$ of tomography results locate within the error range of $\pm 3.75 \times 10^{10} \mathrm{el} / \mathrm{m}^{3}$. The vertical density profiles at different latitudes are further presented in Fig. 6b-d. It can be seen that $h m F 2$ and $N m F 2$ determined by the GPS tomography algorithm are observed at almost the similar altitudes and densities as the OSSE model truth, except the $N m F 2$ at the location of $40^{\circ} \mathrm{N}$ latitude and $140^{\circ} \mathrm{E}$ longitude (Fig. $6 \mathrm{~d}$ ). The reconstructed $h m F 2$ at the low and high latitudes (Fig. 6b, d) is overestimated around $20 \mathrm{~km}$ in altitude between tomography and model truth, which might be due to the vertical resolution at the F peak.

\section{Discussion and conclusions}

Because of the lack of horizontal GPS-TEC observation paths and incomplete path length matrix (aforementioned $A$ matrix), the previous ionospheric tomography

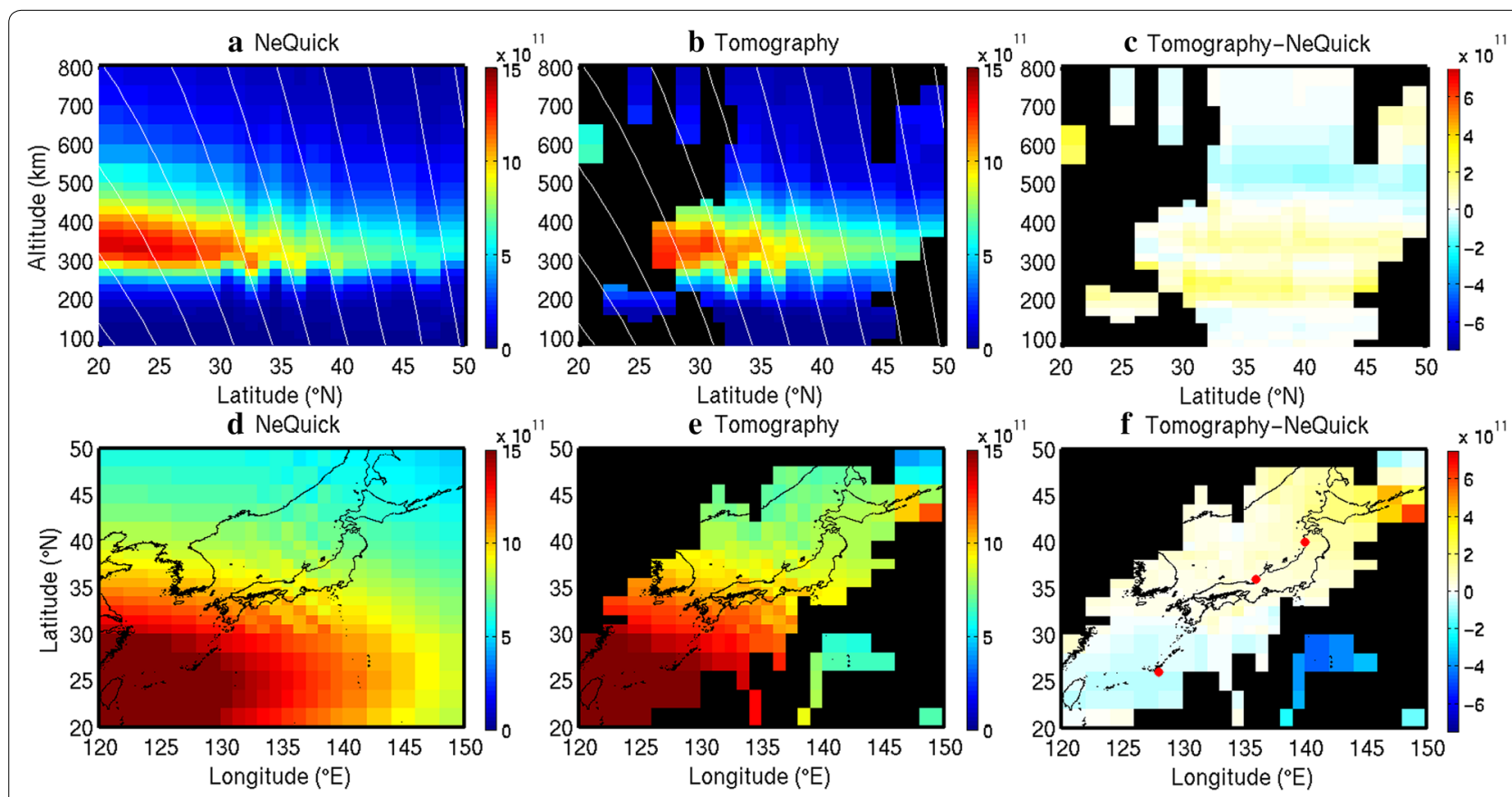

Fig. 5 Same format as Fig. 3 but for the MSTID case. The white lines in (a) and (b) indicate the geomagnetic filed lines. The unit of electron density is $\mathrm{el} / \mathrm{m}^{3}$ 


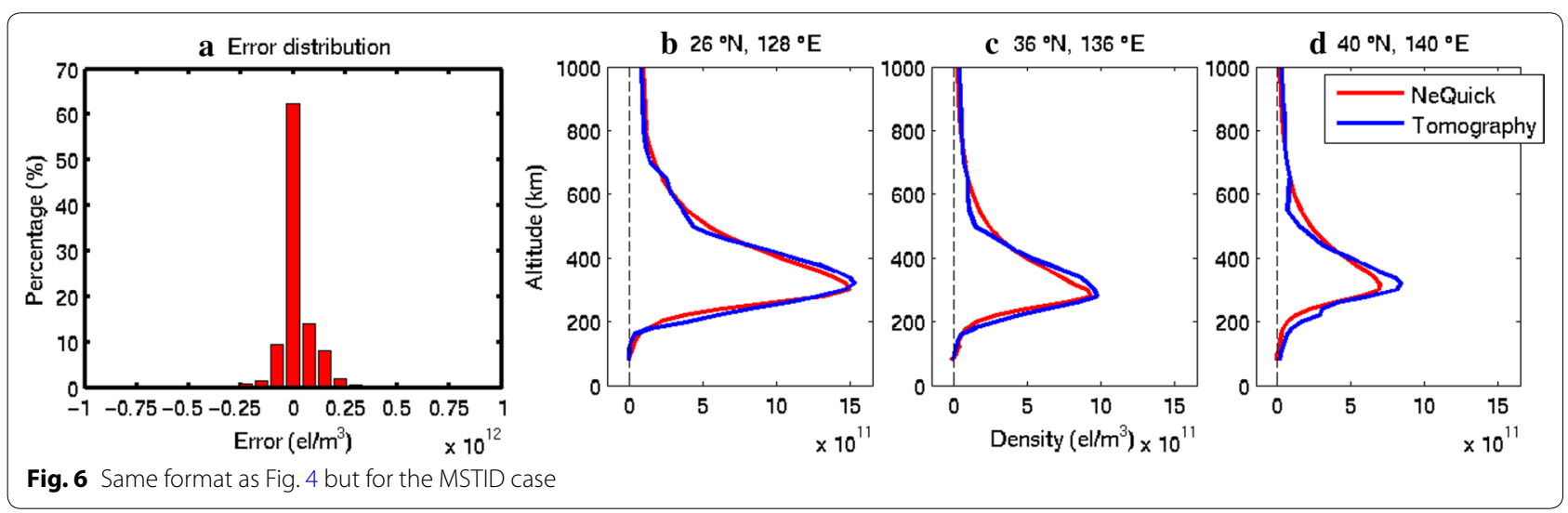

methods, such as algebraic reconstruction technique (ART) (Austen et al. 1986) and multiplicative algebraic reconstruction technique (MART) (Raymund et al. 1990; Ssessanga et al. 2015), usually use the iteration method with an initial guess value to reconstruct the structure of the ionospheric electron density. These iteration methods are a mathematical procedure that generates a sequence of improving approximate solutions for the inverse problems. However, the solution is sensitive to the initial guess value and becomes the same as the initial value for grids without any radio ray path passing by.

In order to resolve the limitations of the GPS tomography, the algorithm of constrained least-square method is developed and the dense ground-based GPS receiver network around Japan region on May 25, 2012, is employed to evaluate its performance. Furthermore, the reliability of this GPS tomography algorithm is validated through the OSSE during the quiet time of ionosphere. This result indicates that an appropriate three-dimensional electron density structure can be reconstructed by using this GPS tomography algorithm with the ground-based GPS-TEC observations. Then, the electron density perturbation structures with $200 \mathrm{~km}$ wavefront above $30^{\circ} \mathrm{N}$ latitudes are further applied to the model truth given by NeQuick for synthetic MSTID structure. The GPS tomography is successfully employed to reconstruct electron density distribution during the disturbed ionosphere. The tomography results have shown that the density perturbation structure can be reconstructed well.

Otsuka et al. (2013) simulated the density structure of MSTID and found the different morphologies for the daytime and nighttime MSTIDs. Although the mechanism of MSTID is still not yet clear, the daytime MSTID might be caused by a gravity wave, while the nighttime MSTID might be generated by the electrodynamical coupling between the Es layer and $\mathrm{F}$ region (Shiokawa et al. 2003; Saito et al. 2007; Otsuka et al. 2007; Seker et al. 2008; Yokoyama et al. 2009). If the MSTID is produced by the electrodynamical coupling effect, a field-aligned structure of density should be observed because the electric field prefers to transport along the magnetic field. In this paper, we simulated the electron density structure of nighttime MSTID by Otsuka et al. (2013) and successfully reconstructed the MSTID density structure by the GPS tomography. It indicates the capability of GPS tomography for the studies of disturbed ionosphere, such as the MSTID. In the future, the developed algorithm of GPS tomography in this paper will be used to reconstruct the three-dimensional electron density structure by the real GPS-TEC observations from GEONET and further provide the near-real-time ionospheric density structure over Japan. In the present version of GPS tomography process, it needs around $30 \mathrm{~min}$ (including the GPS-TEC calculation and the reconstruction times) to reproduce the 3D structure of electron density. A Python version of GPS tomography is ongoing to develop to speed up the whole processes. The first result shows the time can be reduced to around $5 \mathrm{~min}$, which could make the "near real time" possible after the program being completed.

\section{Authors' contributions}

CHC has carried out the GPS tomography method and the program coding and drafted the text. AS has given very important conceptions on the algorithm of GPS tomography. CHL has critically evaluated the text for scientific content and elaborated it. MY, SS, and GKS have contributed well in the improvement of GPS tomography program. All authors read and approved the final manuscript.

\section{Author details}

1 Department of Earth Sciences, National Cheng Kung University, Tainan, Taiwan. ${ }^{2}$ Department of Geophysics, Kyoto University, Kyoto, Japan. ${ }^{3}$ Research Institute for Sustainable Humanosphere, Kyoto University, Kyoto, Japan. ${ }^{4}$ Indian Institute of Geomagnetism, Mumbai, India.

\section{Acknowledgements}

The GPS-TEC data are provided by the Geospatial Information Authority in Japan (GEONET, http://www.gsi.go.jp/ENGLISH/index.html). This paper is supported by Ministry of Science and Technology (MOST) and National Space Organization (NSPO) of Taiwan to National Cheng Kung University under MOST-103-2111-M-006-001-MY2 and NSPO-S-102132.

\section{Competing interests}

The authors declare that they have no competing interests. 


\section{Appendix: Solution of constrained least-square method}

In order to solve the electron density ( $x$ matrix) from the cost function $J(x)$ (Eq. 3), at the first step, we expand Eq. (3) as follows:

$$
\begin{aligned}
J(x) & =(b-A x)^{\mathrm{T}}(b-A x)+\lambda(W x)^{\mathrm{T}}(W x) \\
& =b^{2}+A^{\mathrm{T}} A x^{2}-2 A^{\mathrm{T}} b x+\lambda W^{\mathrm{T}} W x^{2}
\end{aligned}
$$

where the $W$ matrix in Eq. (7) is given as:
Davies K (1990) lonospheric radio. Peter Peregrinus Ltd, London Fehmers G (1994) A new algorithm for ionospheric tomography. In: Kersley $L$ (ed) Proceedings of the International Beacon Satellite Symposium. University of Wales, Aberystwyth, pp 52-55

Fremouw EJ, Secan JA, Howe BM (1992) Application of stochastic inverse theory to ionospheric tomography. Radio Sci 27:721-732

Hunsucker R (1982) Atmospheric gravity waves generated in the high-latitude ionosphere: a review. Rev Geophys Space Phys 20:293-315

Kelley MC (2011) On the origin of mesoscale TIDs at midlatitudes. Ann Geophys 29:361-366

Kersley $L$ (2005) lonospheric tomography and its applications in radio science and geophysical investigations. Ann Geophys 48(3):535-548

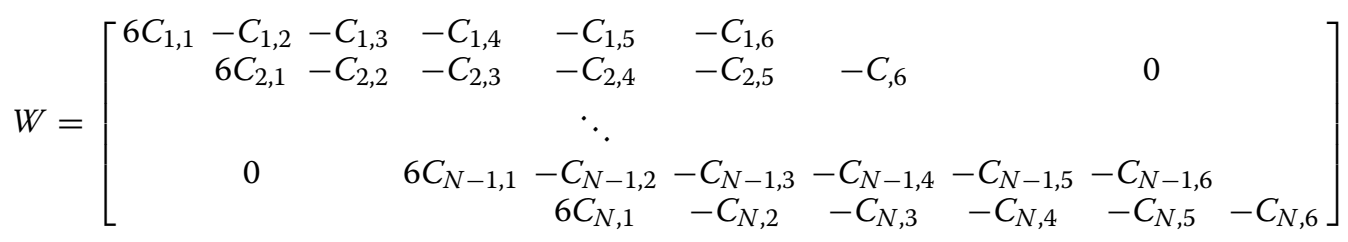

in which $N$ is the total number of observation path and $C_{j k}$ matrix is the constrain parameter depending on the altitude, which is given in Table 1.

At the next step, we assume the partial difference equation of $\partial J(x) / \partial x$ equals 0 to get the optimization solution. Therefore, Eq. (7) can be written as:

$$
\frac{\partial J(x)}{\partial x}=2 A^{\mathrm{T}} A x-2 A^{\mathrm{T}} b+2 \lambda W^{\mathrm{T}} W x=0
$$

Then, we move the second term of Eq. (9) to the right side of equation and can calculate the solution ( $x$ matrix) as follows:

$$
\begin{aligned}
& \left(A^{\mathrm{T}} A+\lambda W^{\mathrm{T}} W\right) x=A^{\mathrm{T}} b \\
& x=\left(A^{\mathrm{T}} A+\lambda W^{\mathrm{T}} W\right)^{-1} A^{\mathrm{T}} b
\end{aligned}
$$

Finally, we can get the result by Eq. (11), which is the same as Eq. (4) in the text.

Received: 1 May 2015 Accepted: 11 February 2016

Published online: 27 February 2016

\section{References}

Andreeva ES, Kunitsyn VE, Tereshchenko ED (1992) Phase difference radio tomography of the ionosphere. Ann Geophys 10:849-855

Austen JR, Franke SJ, Liu CH, Yeh KC (1986) Applications of computerized tomography techniques to ionospheric research. In: International Beacon Satellite Symposium June 9-14, Oulu Finland, Proceeding part 1, pp 25-36

Austen JR, Franke SJ, Liu CH (1988) lonospheric imaging using computerized tomography. Radio Sci 23(3):299-307

Bilitza D, Reinisch B (2008) International reference ionosphere 2007: improvements and new parameters. J Adv Space Res 42(4):599-609

Chen CH (2012) Modeling and observational studies of plasma density anomalies and earthquake-triggered disturbances in the mid-latitude ionosphere, PhD thesis, Department of Geophys., Kyoto University, Kyoto, Japan
Kunitake M, Ohtaka K, Maruyama T, Tokumaru M, Marioka A, Wantabe S (1995) Tomographic imaging of the ionosphere over Japan by the modified truncated SVD method. Ann Geophys 13:1303-1310

Liu JY, Tsai HF, Jung TK (1996) Total electron content obtained by using the global positioning system. Terr Atmos Ocean Sci 7:107-117

Ogawa T, Balan N, Otsuka Y, Shiokawa K, Ihara C, Shimomai T, Saito A (2002) Observations and modeling of $630 \mathrm{~nm}$ air-glow and total electron content associated with traveling ionospheric disturbances over Shigaraki, Japan. Earth Planets Space 54:45-56

Otsuka Y, Onoma F, Shiokawa K, Ogawa T, Yamamoto M, Fukao S (2007) Simultaneous observations of nighttime medium-scale traveling ionospheric disturbances and E-region field-aligned irregularities at midlatitude. J Geophys Res 112:A06317. doi:10.1029/2005JA011548

Otsuka Y, Shiokawa K, Ogawa T, Yokoyama T, Yamamoto M (2009) Spatial relationship of nighttime medium-scale traveling ionospheric disturbances and $F$ region field-aligned irregularities observed with two spaced all-sky airglow imagers and the middle and upper atmosphere radar. J Geophys Res 114:A05302. doi:10.1029/2008JA013902

Otsuka Y, Kotake N, Shiokawa K, Ogawa T, Tsugawa T, Saito A (2011) Statistical study of medium-scale traveling ionospheric disturbances observed with a GPS receiver network in Japan. In: Aeronomy of the earth's atmosphere and ionosphere, IAGA Special Sopron Book Series, vol 2, part 3, pp 291-299. doi:10.1007/978-94-007-0326-1_21

Otsuka YK, Suzuki S, Nakagawa M, Nishioka K Shiokawa, Tsugawa T (2013) GPS observations of medium-scale traveling ionospheric disturbances over Europe. Ann Geophys 31:163-172

Pryse SE (2003) Radio tomography: a new experimental technique. Surv Geophys 24:1-38

Pryse SE, Kersley L (1992) A preliminary experimental test of ionospheric tomography. J Atmos Terr Phys 54:1007-1012

Radicella SM, Leitinger R (2001) The evolution of the DGR approach to model electron density profiles. Adv Space Res 27(1):35-40

Raymund TD (1995) Comparison of several ionospheric tomography algorithms. Ann Geophys 13:1254-1262

Raymund TD, Austen JR, Franke SJ, Lin CH, Klobuchar JA, Sralker J (1990) Application of computerized tomography to the investigation of ionospheric structures. Radio Sci 25(3):771-789

Raymund TD, Franke SJ, Yeh KC (1994) Ionospheric tomography: its limitations and reconstruction methods. J Atmos Phys 56:637-657

Saito A, Fukao S, Miyazaki S (1998) High resolution mapping of TEC perturbations with the GSI GPS network over Japan. Geophys Res Lett 25:3079-3082

Saito S, Yamamoto M, Hashiguchi H, Maegawa A, Saito A (2007) Observational evidence of coupling between quasi-periodic echoes and medium scale traveling ionospheric disturbances. Ann Geophys 25:2185-2194. doi:10.5194/angeo-25-2185-2007 
Sardon E, Rius A, Zarraoa N (1994) Estimation of the transmitter and receiver differential biases and the ionospheric total electron content from Global Positioning System observations. Radio Sci 29:577-586

Seemala GK, Yamamoto M, Saito A, Chen CH (2014) Three-dimensional GPS ionospheric tomography over Japan using constrained least squares. J Geophys Res Space Phys 119:3044-3052. doi:10.1002/2013JA019582

Seker I, Livneh DJ, Makela JJ, Mathews JD (2008) Tracking F region plasma depletion bands using GPS-TEC, incoherent scatter radar, and all-sky imaging at Arecibo. Earth Planets Space 60:633-646

Shiokawa K, Ihara C, Otsuka Y, Ogawa T (2003) Statistical study of nighttime medium-scale traveling ionospheric disturbances using midlatitude airglow imagers. J Geophys Res 108:1052. doi:10.1029/2002JA009491

Ssessanga N, Kim YH, Kim E (2015) Vertical structure of medium-scale traveling ionospheric disturbances. Geophys Res Lett 42:9156-9165. doi:10.1002/ 2015GL066093
Tsugawa T, Saito A, Otsuka Y (2004) A statistical study of large-scale traveling ionospheric disturbances using the GPS network in Japan. J Geophys Res 109:A06302. doi:10.1029/2003JA010302

Wilson DB, Mannucci AJ, Edwards CD, Roth T (1992) Global ionospheric maps using a global network of GPS receivers, In: The Interantional Beacon Satellite Symposium, MIT, Cambridge, MA, July 6-12

Yeh KC, Raymund TD (1991) Limitations of ionospheric imaging by tomography. Radio Sci 26(6):1361-1380. doi:10.1029/91RS01873

Yokoyama T, Hysell D, Otsuka Y, Yamamoto M (2009) Three-dimensional simulation of the coupled Perkins and Es-layer instabilities in the nighttime midlatitude ionosphere. J Geophys Res 114:A03308. doi:10.1029/20 08JA013789

\section{Submit your manuscript to a SpringerOpen ${ }^{\circ}$ journal and benefit from:}

- Convenient online submission

- Rigorous peer review

- Immediate publication on acceptance

- Open access: articles freely available online

- High visibility within the field

- Retaining the copyright to your article

Submit your next manuscript at $\downarrow$ springeropen.com 